\title{
Radiation Enhances the Epithelial- Mesenchymal Transition of A549 Cells via miR3591-5p/USP33/PPM1A
}

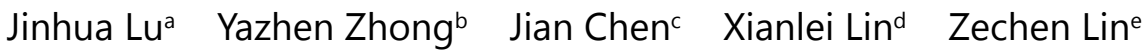 \\ Nan Wang ${ }^{f}$ Shengyou Lin ${ }^{d}$
}

aDepartment of Traditional Chinese Comprehensive Medical Oncology, Hangzhou Cancer Hospital, Hangzhou Hospital affiliated to Nanjing Medical University, Hangzhou Cancer Center, Hangzhou, bepartment of Integrated Chinese and Western Medicine, Hangzhou Cancer Hospital, Hangzhou Hospital affiliated to Nanjing Medical University, Hangzhou Cancer Center, Hangzhou, 'The First Affiliated Hospital of Zhejiang Chinese Medicine University, Hangzhou, dDepartment of Oncology, Guangxing Hospital Affiliated to Zhejiang Chinese Medical University, Hangzhou, eThe Fourth Clinical Medical College, Zhejiang Chinese Medical University, Hangzhou, 'The Second Clinical Medical College, Zhejiang Chinese Medical University, Hangzhou, China

\section{Key Words}

Radiotherapy $\bullet$ Lung cancer $\bullet$ miRNA $\bullet$ Ubiquitination

\begin{abstract}
Background/Aims: Radiotherapy plays a critical role in lung cancer treatment. Radiation can activate transforming growth factor- $\beta$ (TGF- $\beta$ ) signaling and induce the epithelialmesenchymal transition (EMT), which may lead to distant metastases. MicroRNAs (miRNAs) have been suggested to affect radiotherapy in lung cancer. Methods: miRNA Next-Generation Sequencing was performed to investigate the effects of irradiation on the miRNA profile of lung cancer A549 cells. The functions of identified miRNA on the radiation induced EMT and TGF- $\beta$ activation in A549 cells were then explored. Protein expression was evaluated by western blotting. Immunofluorescence staining was performed to detect the localization of Snail. Luciferase Assay was used to determine the target gene regulated by the identified miRNA. Results: Radiation time-dependently induced EMT in A549 lung cancer cells as indicated by the changes of morphology, the expression of EMT marker proteins (E-cadherin, $\alpha-S M A$ and Vimentin) and the nuclear localization of Snail. Moreover, miR3591-5p was identified as the most significant increased miRNA in response to radiation, and further experiments indicated that miR-3591-5p was required for radiation induced EMT and TGF- $\beta$ / Smad2/3 activation. Ubiquitin Specific Peptidase 33 (USP33) was a downstream target of miR-3591-5p as predicted by TargetScan and validated by $3^{\prime}$ untranslated regions (UTRs) Luciferase Assay. USP33 could deubiquitinate PPM1A (protein phosphatase, Mg2+/Mn2+ dependent $1 \mathrm{~A}$ ), a phosphatase for Smad2/3. Ectopic expression of USP33 or PPM1A partially J. Lu and $\mathrm{Y}$. Zhong contributed equally to this work.

$\begin{array}{ll}\text { Shengyou Lin } & \text { Department of Oncology, GuangXing Hospital Aff. to Zhejiang Chinese Med.Univ. } \\ & 453 \text { Tiyuchang Road, Xihu District, Hangzhou 310007 (China) } \\ & \text { Tel. +86-57185827882, E-Mail linsy0628@126.com }\end{array}$
\end{abstract}


Lu et al.: EMT via miR3591-5p/USP33/PPM1A

abolished the effects of miR-3591-5p on EMT and TGF- $\beta$ / Smad2/3 activation. Conclusion: The present study revealed the critical role of miR-3591-5p/USP33/PPM1A in radiationinduced EMT via TGF- $\beta$ signaling and may suggest novel radiation sensitise strategies for lung cancer.

\section{Introduction}

Lung cancer is one of the most frequent cancers worldwide. It is a leading cause of death from cancer due to the high mortality rates [1]. Lung cancer is broadly classified into small cell lung cancer (SCLC) and non-small cell lung cancer (NSCLC), and NSCLC accounts for $85 \%$ cases of lung cancer [2]. Much scientific advances have been made in the field of lung cancer treatment, such as surgical therapy, chemotherapy and radiotherapy, over the past few decades. The improvement in the use of these therapy has enhanced the disease-free survival of lung cancer survivors [3]. However, many challenges still remain to be overcome in lung cancer treatment. For instance, although radiation therapy plays a critical role in cancer treatment, the efficacy is limited in many cases [4, 5]. Previous researchers have observed distant metastases in many patients with locally advanced lung cancer receiving conventional radiotherapy [6]. Growing evidence has suggested that epithelial-mesenchymal transition (EMT) plays a central role in metastasis [7]. During EMT, epithelial cells undergoes morphological changes from cobblestone phonotype to elongated fibroblastic phonotype. EMT is characterized by the downregulation of epithelial molecular markers such as E-cadherin and the upregulation of mesenchymal molecular markers such as Vimentin and $\alpha$-smooth muscle actin $(\alpha-S M A)[7,8]$.

Various regulatory networks and extracellular stimuli, including transforming growth factor- $\beta$ (TGF- $\beta$ ), hypoxia and radiation [9], have been identified to regulate EMT. In A549 lung cancer cells, radiation can induce the EMT via the activation of TGF- $\beta$ signaling $[10,11]$. A crucial step in TGF- $\beta$ signaling is the phosphorylation and activation of Smad2/3 by TGF- $\beta$ receptor. PPM1A (protein phosphatase, Mg2+/Mn2+ dependent $1 \mathrm{~A}$ ), a member of the PP2C family of serine/threonine protein phosphatase, has been reported to dephosphorylate $S m a d 2 / 3$ to terminate TGF- $\beta /$ Smad signaling [12-14]. PPM1A overexpression abolished the antiproliferative and transcriptional responses of TGF- $\beta$ [12]. Knockdown of PPM1A promoted the invasion and EMT in bladder cancer cells via the activation of TGF- $\beta$ /Smad signaling pathway [14]. Several serine/threonine protein phosphatases, including PPM1D, PP1 and PHLPPL have been linked to the radiation response $[15,16]$, while it is not known whether PPM1A involves in radiation response of lung cancer cells.

microRNAs (miRNAs) are a class of small noncoding RNAs that function as negative regulators of gene expression at the post-transcriptional level. miRNAs interact with the 3' untranslated regions (UTR) of their target mRNAs, which leads to the degradation of target mRNAs or translational repression [17]. Target mRNAs of human miRNAs involve in tumorigenesis processes, such as cell differentiation, proliferation, apoptosis and metastasis, including lung cancer [18-21]. Furthermore, expression of some miRNAs has been linked to radiotherapy in lung cancer [22-25].

In the current study, miRNA Next-Generation Sequencing was used to investigate the effects of irradiation on the miRNA profile of A549 cells. miR-3591-5p, the most significant increased miRNA in response to radiation, was found required for radiation induced EMT and TGF- $\beta$ activation. Ubiquitin Specific Peptidase 33 (USP33), as a downstream of miR-3591$5 p$, could deubiquitinate PPM1A. Ectopic expression of USP33 or PPM1A partially abolished the effects of miR-3591-5p on EMT and TGF- $\beta$ activation. Our findings demonstrate the role of miR-3591-5p/USP33/PPM1A in radiation-induced EMT via TGF- $\beta$ signaling and may suggest novel radiation sensitise strategies for lung cancer. 


\section{Cellular Physiology Cell Physiol Biochem 2018;50:721-733 and Biochemistry Published online: 12 October 2018 \begin{tabular}{l|l} 
DOI: 10.1159/000494238 2018 The Author(s). Published by S. Karger AG, Basel \\
www.karger.com/cpb
\end{tabular}}

Lu et al.: EMT via miR3591-5p/USP33/PPM1A

\section{Materials and Methods}

\section{Cell culture and radiation treatment}

A549 cells (cell bank of Shanghai Biology Institute, Chinese Academy of Science; Shanghai, China) were grown in Dulbecco's modified Eagle's medium (Hyclone, Logan, UT, USA) supplemented with $10 \%$ fetal bovine serum (Invitrogen, Carlsbad, $\mathrm{CA}$ ) at $37^{\circ} \mathrm{C}$ with $5 \% \mathrm{CO}_{2}$. The cells were radiation with $8 \mathrm{~Gy}$ of ${ }^{60} \mathrm{Co} \gamma$-rays (Hangzhou Cancer Hospital, dose rate: $3.64 \mathrm{~Gy} / \mathrm{min}$ ) as previously reported [26, 27].

\section{Western blotting analysis}

The cells were lysed in ice-cold radioimmunoprecipitation buffer (RIPA, Solarbio, Beijing, China) and clarified by centrifugation at 12, $000 \mathrm{~g}$ for $20 \mathrm{~min}$. After separating on $10 \%$ or $15 \%$ SDS-PAGE gels, western blotting analysis was performed with primary antibodies, corresponding horseradish peroxidase-labelled secondary antibodies, and enhanced chemiluminescence substrate mixture (Millipore, Bredford, MA, USA) as previously described [27]. The experiments were repeated for three times with GAPDH as loading control. The sources of primary antibodies are as follows: anti-E-cadherin (Cell Signaling Technology, Danvers, MA, USA; diluted 1:1000), anti- $\alpha$-SMA (Abcam, Cambridge, MA, USA; diluted 1:500), anti-Vimentin (Cell Signaling Technology; diluted 1:1000), anti-phosphor-Smad2 (Abcam; diluted 1:800), anti-Smad2 (Abcam; diluted 1:1000), anti-phosphor-Smad3(Abcam; diluted 1:500), anti-Smad3 (Abcam; diluted 1:1000), antiUSP33 (Abcam; diluted 1:2000), anti-PPM1A (Cell Signaling Technology; diluted 1:1000) and anti-GAPDH (Cell Signaling Technology; diluted 1:2000).

\section{Immunofluorescence analysis}

At $24 \mathrm{~h}$ or $48 \mathrm{~h}$ post irradiation, immunofluorescence analysis was performed on cells cultured on coverslips as previously described [27]. In brief, the cells were fixed with 4\% paraformaldehyde for $15 \mathrm{~min}$, permeabilized with $0.05 \%$ Triton X-100 for 10 min, blocked with $5 \%$ goat serum for 1 hour and probed with anti-Snail (Abcam; diluted 1:100) for 1 hour followed by Alexa Fluor ${ }^{8} 488$ donkey anti-rabbit IgG antibody (Cell Signaling Technology, diluted 1:500) for 1 hour in the dark. The nuclear was stained with 4, 6-diamino2-phenylindole (DAPI; Beyotime). Images were viewed and collected under a fluorescence microscope.

\section{RNA extraction and next-generation sequencing}

At $48 \mathrm{~h}$ post irradiation, total RNA was extracted from A549 cells with or without radiation treatment using TRIzol reagent (Invitrogen) as per the manufacturer's instructions. After removing any contaminating genomic DNA with DNase, small RNA library was constructed and then sequenced on Illumina HiSeq sequencing systems (Illumina, San Diego, CA, USA). Raw data were cleaned with Illumina's Genome Analyzer Pipeline software, and then aligned against microRNA database (miRBASE release $21 \mathrm{at} \mathrm{http://}$ www.mirbase.org/). Two-tailed Student's t-test was used to analyze differences in miRNA expression levels between the radiation and control groups. Significant differences were set at fold change $\geq 2.0$ and adjusted $\mathrm{p}$-value (q value) $<0.05$.

\section{Quantitative RT-PCR (qRT-PCR)}

Total RNA was reversed transcribed into first-strand cDNA with M-MLV Reverse Transcriptase Kit (Thermo Fisher Scientific, Rockford, IL, USA). The synthesized cDNA was used as template for qPCR analysis with SYBR Green qPCR Master Mix (Thermo Fisher Scientific) on ABI PRISM 7300 Sequence Detection System (Applied Biosystem, Foster City, CA, USA). The PCR primers are as follows: USP33, 5'ATCTGTTGTGCCTACTACTC-3' (forward primer) and 5'- GCTCTTGTCTTCTTCCATTG-3' (reversed primer); PPM1A, 5'-ATGAGTCAGCACTGCCATAC-3' (forward primer) and 5'-CCCACTATTACCTCCAGGAATC-3' (reversed primer); GAPDH, 5'-CACCCACTCCTCCACCTTTG-3' (forward primer) and 5'- CCACCACCCTGTTGCTGTAG-3' (reversed primer). The ${ }^{2-\Delta \Delta} \mathrm{Ct}$ method [28] was used to calculate the mRNA expression of target genes with GAPDH as an internal control.

miRNA-3591-5p expression was analyzed by stem-loop real-time RT-PCR with U6 RNA as an internal control. First, extracted RNA was converted into cDNAs with stem-loop reverse transcription primers (miR-3591-5p, 5'- CTCAACTGGTGTCGTGGAGTCGGCAATTCAGTTGAGTCAAAC-3'; U6, 5'CACCACGTTTATACGCCGGTG -3') with cDNA synthesis kit (Thermo Fisher Scientific). Second, qPCR analysis was then performed using SYBR Green qPCR Master Mix (Thermo Fisher Scientific) with miR-3591-5p 


\section{Cellular Physiology Cell Physiol Biochem 2018;50:721-733 \begin{tabular}{l|l|l} 
and Biochemistry & DOI:1159/000494238 & $\begin{array}{l}\text { (c) 2018 The Author(s). Published by S. Karger AG, Basel } \\
\text { www.karger.com/cpb }\end{array}$
\end{tabular}}

Lu et al.: EMT via miR3591-5p/USP33/PPM1A

or U6 -specific forward primer (miR-3591-5p, 5'- ACACTCCAGCTGGG TTTAGTGTGATAATGGC-3'; U6, 5'-CTCGCTTCGGCAGCACA-3') and a universal reverse primer (5'-TGGTGTCGTGGAGTCG-3').

Transfection of miR-3591-5p mimic and inhibitor

miR-3591-5p mimic (mim, 5'-UUUAGUGUGAUAAUGGCGUUUGA-3'), miR-3591-5p inhibitor (inh, 5'- UCAAACGCCAUUAUCACACUAAA-3'), control mimic (NC-mim, 5'-UUGUACUACACAAAAGUACUG-3') and control inhibitor (NC-inh, 5'-CAGUACUUUUGUGUAGUACAA-3') were synthesized by Genepharm Technologies (Shanghai, China) and transfected into A549 cells with lipofectamine 2000 reagent (Invitrogen) following the manufacturer's instructions.

Enzyme-linked immunosorbent assay (ELISA)

The culture medium were collected $8 \mathrm{~h}$ after irradiation and subjected to ELISA analysis as previously described [27].

USP33 and PPM1A overexpression

The full-length human USP33 and PPM1A cDNAs were inserted into EcoRI and BamHI sites of the expression vector pLVX-puro (Clontech, Palo Alto, CA, USA) and confirmed by DNA sequencing. The recombinant lentivirus of overexpressed USP33 (USP33 OE)/ PPM1A (PPM1A OE), and control lentivirus (Vector) were prepared by transfection into 293T cells as described previously [29].

Luciferase assay

The full-length 3'UTR of USP33 was inserted into pGL3 Vector (Promega, Madison, WI). A549 cells were co-transfected with pGL3-USP33 plasmid, pRL-CMV plasmid (Promega) and miR-3591-5p mimic/ inhibitor by using lipofectamine 2000 reagent (Invitrogen). Dual-luciferase reporter assay (Promega) was performed at $48 \mathrm{~h}$ post transfection. The luciferase activity was normalized to Ranilla luciferase and then to a control sample transfected with mimic control or inhibitor control.

\section{Immunoprecipitation}

The proteins were extracted from indicated cells with RIPA buffer. The cell lysate was incubated with anti-PPM1A (Cell Signaling Technology) or control IgG at $4{ }^{\circ} \mathrm{C}$ for $2 \mathrm{~h}$, and then with protein A agarose beads (Roche, Indianapolis, IN, USA) at $4{ }^{\circ} \mathrm{C}$ for $1 \mathrm{~h}$. The immunoprecipitated complexes were washed 3 times with RIPA buffer, boiled in sample buffer, and then subjected to western blot analysis with anti-ubiquitin (Abcam).

\section{Statistical analysis}

The data were presented as the mean \pm standard deviation (SD) of three independent experiments. Statistical analysis was done using Graphpad Prism software version 6.0 (GraphPad, San Diego, CA, USA). The statistical significant differences were estimated by one-way analysis of variance (ANOVA) followed by post hoc Tukey's test. A $P$ value less than 0.05 was considered statistically significant.

\section{Results}

Radiation induced the changes of morphology and the expression of EMT-related proteins A549 cells were subjected to radiation and cellular morphology was observed after cultured for $24 \mathrm{~h}$ or $48 \mathrm{~h}$. As illustrated in Fig. 1A, control A549 cells without radiation treatment (Mock) had a classical epithelial morphology, and cells with radiation treatment displayed an elongated spindle-like morphology. In addition, the effects of radiation treatment on the expression of EMT-related proteins was also assessed by western blotting. The results showed that radiation exposure caused a significant reduction in E-cadherin expression and a remarkable increase in the expression of Vimentin and $\alpha$-SMA (Fig. 1B). Snail localized in the nucleus is active to repress E-cadherin expression [8]. Further, immunofluorescence analysis was performed to assess the expression and nucleus location of Snail (Fig. 1C). Radiation exposure remarkably enhanced the nucleus signal of Snail (green fluorescence). All 


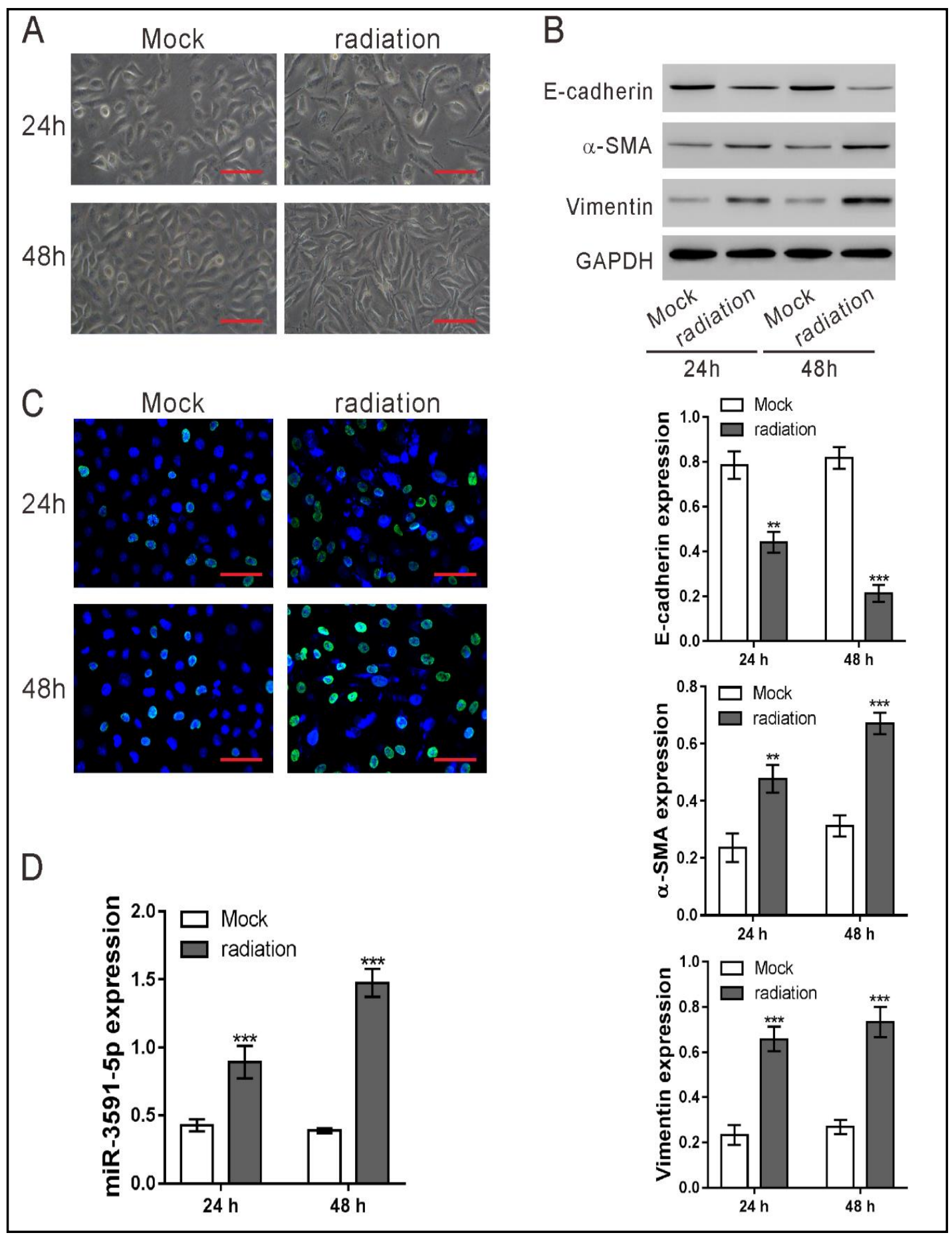

Fig. 1. Radiation induced EMT and miR-3591-5p expression in A549 cells. A549 cells were irradiation with $8 \mathrm{~Gy}$ and then cultured for $24 \mathrm{~h}$ and $48 \mathrm{~h}$. Control cells did not receive radiation treatment (Mock). (A) Cell morphology was observed under a light microscope (Scale bar: $50 \mathrm{~m}$ ). (B) Western blot analysis was performed to detect the expression of E-cadherin, Vimentin and $\alpha$-SMA. Representative blots and quantification for three consecutive data are shown. (C) The expression and nucleus location of Snail was assessed by immunofluorescence staining (green). The nuclear was stained with DAPI (blue) (Scale bar: 50 $\mu \mathrm{m})$. Representative images from three independent experiments are shown. (D) The expression of miR3591-5p was detected by qRT-PCR. ${ }^{* * *} \mathrm{P}<0.001$ versus Mock. 
the above effects of radiation were in a time-dependent manner. Our data suggested that radiation induced EMT of A549 cells.

\section{Differentially expressed miRNAs (DEMs) in response to radiation}

To investigate the effects of irradiation on the miRNA profile of A549 cells, radiationtreated A549 cells were cultured for $48 \mathrm{~h}$, and RNA was extracted and then subjected to miRNA Next-Generation Sequencing. miRNAs with a fold change $\geq 2.0$, and $q$ value $<0.05$ were identified as DEMs. We identified 24 significantly DEMs, including 10 up-regulations and 14 down-regulation (Table 1), in radiation-treated cells compared with control cells. miR-3591-5p was the most significant DEMs. qRT-PCR results validated the up-regulated of miR-3591-5p in radiation-treated A549 cells (Fig. 1D).

\section{Functions of miR-3591-5p in radiation-treated A549 cells}

To check whether miR-3591-5p involved in radiation-induced EMT, this miRNA was overexpressed and inhibited in A549 cells by transfection with mimic and inhibitor, respectively. The cells were then subjected to radiation exposure $24 \mathrm{~h}$ later and cultured for another $48 \mathrm{~h}$. As shown in Fig. 2A, radiation-induced miR-3591-5p expression was significantly blocked by the inhibition of miR-3591-5p, and remarkably promoted by the overexpression of miR-3591-5p. Decreased expression of miR-3591-5p significantly reversed the effect of radiation on cell morphology changes (Fig. 2B) and the expression of EMT-related protein (Fig. 2C), while increased expression of miR-3591-5p showed the reverse effects.

\section{Downregulation of miR-3591-5p inactivates TGF- $\beta /$ Smad pathway}

The TGF- $\beta /$ Smad signaling pathway is an important regulator for radiation-induced EMT [10, 11]. To explore the potential molecular mechanisms how miR-3591-5p affected radiationinduced EMT, we assessed the activation of TGF- $\beta /$ Smad pathway by measuring TGF- $\beta$ concentrations in cultured medium and the phosphorylation of Smad2/3. As shown in Fig. $3 \mathrm{~A}$, irradiation caused a significant increase in the TGF- $\beta$ concentrations, which was reduced by the transfection of miR-3591-5p inhibitor and increased by the transfection of miR-3591$5 \mathrm{p}$ mimic. The results of western blotting showed that the levels of phospho-Smad2/3 was downregulated in miR-3591-5p inhibitor-transfected A549 cells compared to that in control inhibitor-transfected A549 cells after radiation (Fig. 3B). Reverse effects were observed in miR-3591-5p mimic-transfected A549 cells. This suggested that activation of TGF- $\beta / \mathrm{Smad}$ pathway by radiation in A549 cells was suppressed by downregulation of miR-3591-5p. 


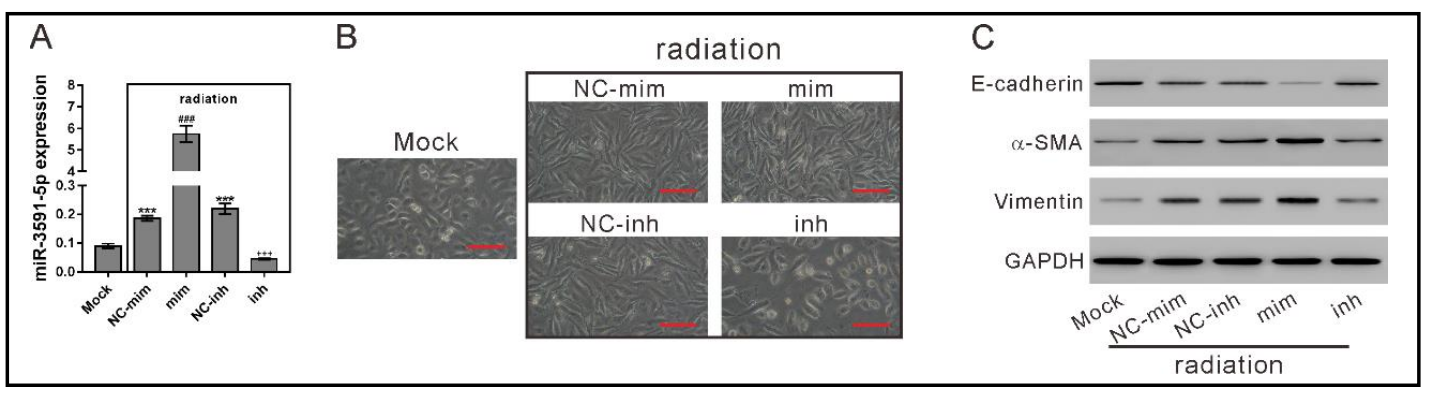

Fig. 2. Functions of miR-3591-5p in radiation-treated A549 cells. A549 cells were transfected with miR3591-5p mimic (mim), miR-3591-5p inhibitor (inh), control mimic (NC-mim) or control inhibitor (NCinh), subjected to radiation exposure $24 \mathrm{~h}$ later, and cultured for another $48 \mathrm{~h}$. Control cells did not receive radiation treatment (Mock). (A) The expression of miR-3591-5p was detected by qRT-PCR. ${ }^{* * *} \mathrm{P}<0.001$ versus Mock; \#\#\#P<0.001 versus NC-mim; $+++\mathrm{P}<0.001$ versus NC-inh. (B) Cell morphology was observed with a light microscope (Scale bar: $50 \mu \mathrm{m}$ ). (C) Protein levels of E-cadherin, Vimentin and $\alpha$-SMA were tested by western blot analysis. Representative images from three independent experiments are shown.

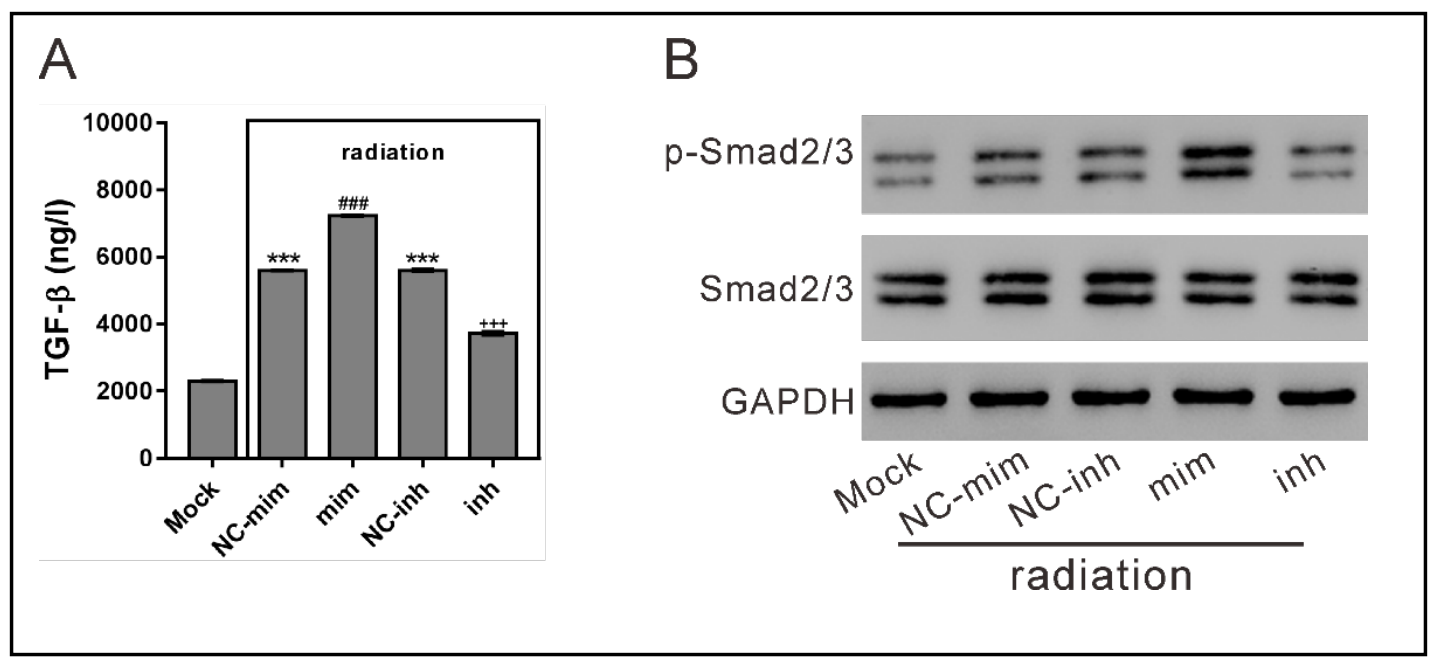

Fig. 3. Downregulation of miR-3591-5p inactivates TGF- $\beta /$ Smad pathway. A549 cells were treated as described in Fig. 2. (A) At $8 \mathrm{~h}$ post radiation, the concentration of TGF- $\beta$ in the cultured medium was evaluated by ELISA. ${ }^{* * *} \mathrm{P}<0.001$ versus Mock; \#\#\#P<0.001 versus NC-mim; $+++\mathrm{P}<0.001$ versus NC-inh. (B) At $8 \mathrm{~h}$ post irradiation, the phosphorylated and total forms of Smad2/3 was detected by western blot analyses with GAPDH as loading control. Representative images from three independent experiments are shown.

\section{USP33 is a potential target of miR-3591-5p}

To investigate the downstream targets of miR-3591-5p, TargetScan (http://www. targetscan.org/) was used and we found that miR-3591-5p could target USP33. USP33 3' untranslational region (UTR) presented a potential binding site of miR-3591-5p (Fig. 4A). qRT-PCR analysis showed that USP33 mRNA expression was significantly inhibited by miR3591-5p mimic, and improved by miR-3591-5p inhibitor in A549 cells (Fig. 4B). Further, 3'UTR luciferase assay indicated that the relative luciferase activity of USP33 3'UTR was also inhibited by miR-3591-5p mimic and enhanced by miR-3591-5p inhibitor (Fig. 4C). These data suggested the direct interaction between miR-3591-5p and USP33 3'UTR caused the decreased expression of USP33 mRNA. 


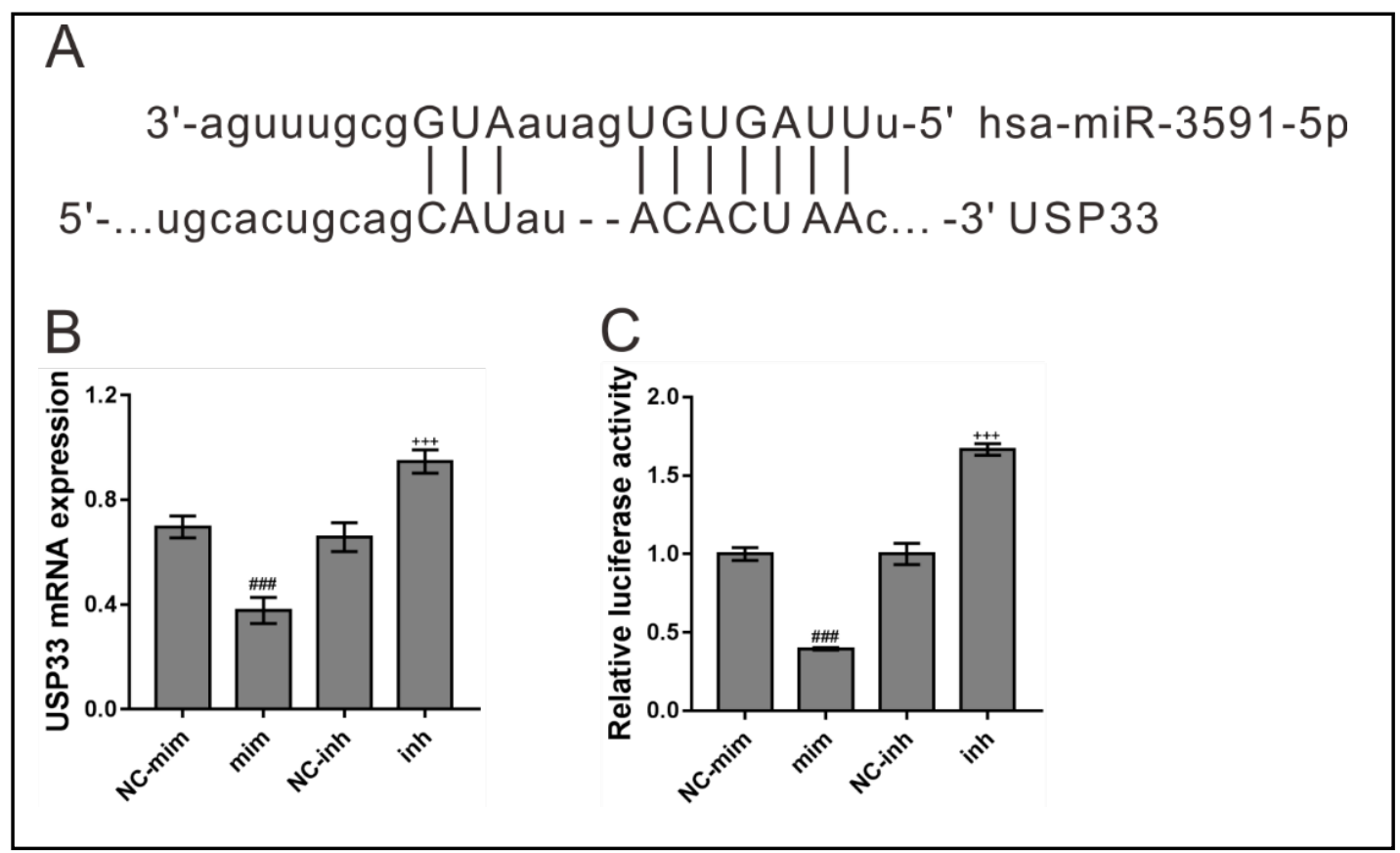

Fig. 4. USP33 is a potential target of miR-3591-5p. (A) Predicted binding sequence of miR-3591-5p in 3'UTR of USP33. (B) A549 cells were transfected with miR-3591-5p mimic (mim), miR-3591-5p inhibitor (inh), control mimic (NC-mim) or control inhibitor (NC-inh). At $48 \mathrm{~h}$ post transfection, USP33 mRNA expression was detected by qRT-PCR. (C) Luciferase assay was performed in A549 cells transfected with USP33 3'UTR and indicated miRNA. \#\#\#P<0.001 versus NC-mim; $+++\mathrm{P}<0.001$ versus $\mathrm{NC}$-inh.

\section{USP33 deubiquitinates PPM1A}

PPM1A can inactivate TGF- $\beta$ /Smad signaling by dephosphorylating Smad2/3 [12-14]. We then explored the effects of USP33 on the expression of PPM1A in A549 cells. As shown in Fig. 5A, USP33 overexpression significantly increased PPM1A protein expression. qRT-PCR analysis showed that PPM1A mRNA expression was not affected by USP33 overexpression (Fig. 5B). Considering that USP33 was a deubiquitinating enzyme, we speculated that PPM1A protein was a substrate of USP33 and USP33 could stabilize PPM1A. As shown in Fig. 5C, radiation treatment significantly increased PPM1A ubiquitination, which was suppressed by the ectopic expression of USP33. These data suggest that USP33 deubiquitinated and stabilized PPM1A in radiation-treated A549 cells.

\section{Overexpression of USP33 or PPM1A blocks the effects of miR-3591-5p}

To test whether USP33 and PPM1A were involved in the functions of miR-3591-5p, we overexpressed USP33 and PPM1A in A549 cells with miR-3591-5p overexpression. The protein expression of PPM1A was verified by western blot analyses (Fig. 6A). miR-3591-5p overexpression significantly decreased E-cadherin expression, and increased the levels of Vimentin and $\alpha$-SMA (Fig. 6B), the release of TGF- $\beta$ (Fig. 6C) and the phosphorylation of Smad2/3 (Fig. 6D). Ectopic expression of USP33 or PPM1A partially abolished the effects of miR-3591-5p on the expression of EMT-related protein and the activation of TGF- $\beta / \mathrm{Smad}$ pathway. 


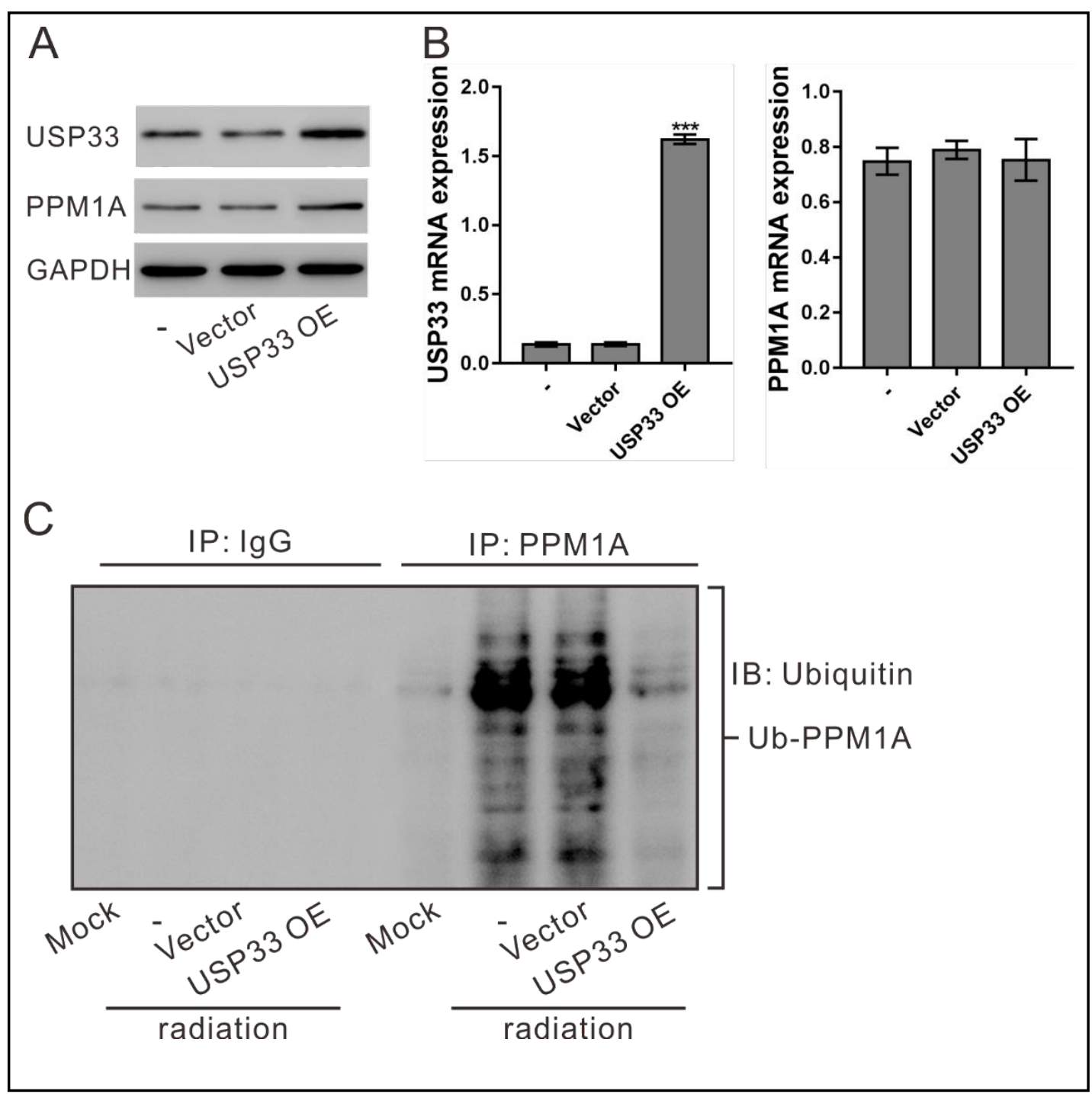

Fig. 5. USP33 deubiquitinates PPM1A. (A, B) A549 cells were transduced with the recombinant lentivirus of overexpressed USP33 (USP33 OE) or control lentivirus (Vector). After $48 \mathrm{~h}$, protein and mRNA expression of USP33 and PPM1A was evaluated by western blot (A) and qRT-PCR analysis, respectively. (C) A549 cells were transduced with USP33 OE or Vector, subjected to radiation exposure $24 \mathrm{~h}$ later, and cultured for another $48 \mathrm{~h}$. Cells without treatment were served as control (Mock). Immunoprecipiation was performed with anti-PPM1A or control IgG, and the ubiquitinated PPM1A was detected by western blot analysis with anti-ubiquitin. 
Fig. 6. Overexpression of USP33 or PPM1A blocks the effects of miR-3591-5p. (A) Lentivirus of overexpressed PPM1A (PPM1A OE) significantly increased PPM1A expression in A549 cells at 48 $\mathrm{h}$ after virus transduction. (B, C, D) A549 cells were treated with miR-3591-5p mimic (mim)/control mimic (NC$\mathrm{mim}$ ) and lentivirus of USP33 OE/PPM1A OE/ Vector. At 48 $\mathrm{h}$ after treatment, expression of EMT-related proteins (B), TGF- $\beta$ concentration in the cultured medium (C) and phospho-Smad2/3 (D) were analyzed. ${ }^{* * *} \mathrm{P}<0.001$ versus NC-mim+Vector; \#\#\#P<0.001 versus mim+Vector.

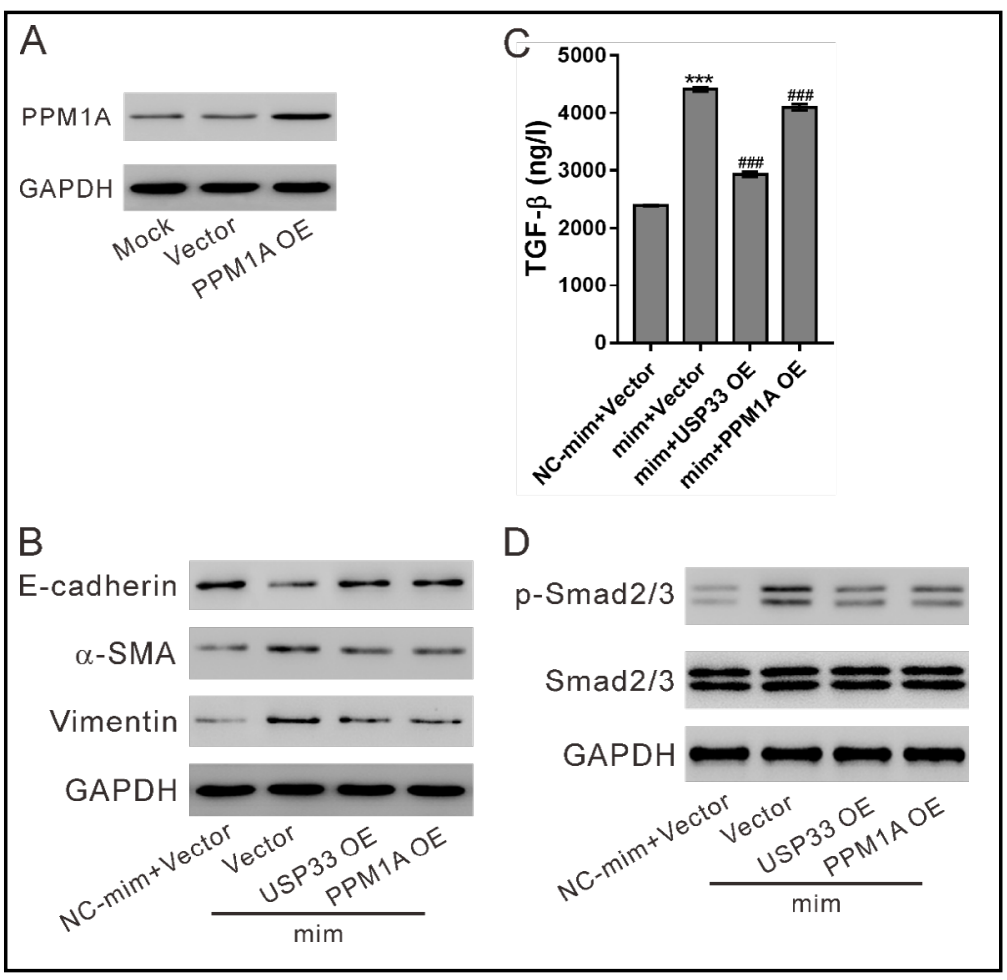

\section{Discussion}

Although radiotherapy plays a crucial role in cancer treatment, many patients had relapse and distant metastases due to radioresistance [6]. Accumulated evidence has demonstrated the central role of EMT in metastasis [7]. In this study, we found that radiation time-dependently induced EMT in A549 cells as indicated by the changes of morphology and the expression of EMT marker proteins (Fig. 1). These finding were in line with the previous study [11].

Abnormal expression of miRNAs has been found in human cancers and miRNAs play a substantial role in the pathogenesis and prognosis of human cancers [18-21,30]. Several miRNAs has been linked to radiotherapy in lung cancer [22-25], which prompt us to investigate the effects of irradiation on the miRNA profile of A549 cells by miRNA Next-Generation Sequencing. We identified 24 significantly DEMs in radiation-treated A549 cells compared with control cells (Table 1), many of which were previously identified to affect radiotherapy in lung cancer, such as miR-215, miR-127-3p, miR-16 [25], let-7g [31] and miR-210 [32], and in other types of cancers, such as miR-210 [33, 34] in hepatoma and miR-31 in esophageal cancer [35]. We also identified many additional novel miRNAs which were differentially expressed in response to irradiation, suggesting that the Next-Generation Sequencing is powerful to study expression profiling. Further experiments should be performed to elucidate the functions of these miRNAs.

miR-3591-5p was the most significant DEMs identified in our study. By performing miRNA microarray analysis or miRNA Sequencing, miR-3591-5p was identified significantly upregulated in the serum of patients with glioblastoma [36] and patients with chronic HBV infection compared to normal control [37]. However, its biological functions have not been characterized. In this study, miR-3591-5p inhibitor partially abolished the effects of radiation on morphological change, the expression of mesenchymal molecular markers (Vimentin and $\alpha$-SMA) and epithelial molecular markers (E-cadherin) (Fig. 2) and the activation of TGF- $\beta$ signaling (Fig. 3). Thus, our findings suggest that miR-3591-5p is required for radiation-induced TGF- $\beta$ activation and EMT process. 
Further, we tried to explore the underlying mechanisms. USP33 was a potential target of miR-3591-5p as predicted by TargetScan. The Luciferase Assay demonstrated that the direct binding of miR-3591-5p to the 3' UTR of USP33, which decreased the mRNA expression of USP33 (Fig. 4). The functional significance of USP33 has been investigated in breast, colon and lung cancer cells. USP33 can inhibit the migration of these cells by its deubiquitinating activity on Robo [38-40]. Here, we found that USP33 could deubiquitinate and stabilize PPM1A (Fig. 5), which has been reported as a phosphatase for Smad2/3 [12-14]. The effects of miR-3591-5p on the expression of EMT-related protein and TGF- $\beta /$ Smad signaling was partially abolished by ectopic expression of USP33 or PPM1A (Fig. 6). Thus, we speculate that radiation up-regulated miR-3591-5p inhibited USP33 transcription, which increased the ubiquitination of PPM1A and then terminated TGF- $\beta$ signaling.

\section{Conclusion}

In conclusion, the findings of our study support the significant contribution of miR3591-5p inhibition on the suppression of radiation-induced EMT. USP33 transcription, PPM1A ubiquitination and TGF- $\beta$ signaling activation is mechanistically implicated in the action of miR-3591-5p inhibition. Increasing understanding of the role of miR-3591-5p/ USP33/PPM1A in radiation-induced EMT may help to improve the efficacy treatments of radiotherapy.

\section{Acknowledgements}

This study was supported by Zhejiang Provincial Natural Science Foundation of China (LY15H290002, LY15H270004), National Natural Science Foundation of China (81173246) and Science Technology Department of Zhejiang Province (2018C03025).

\section{Disclosure Statement}

The authors declare that they have no competing interests.

\section{References}

1 Torre LA, Bray F, Siegel RL, Ferlay J, Lortet-Tieulent J, Jemal A: Global cancer statistics, 2012. CA Cancer J Clin 2015;65:87-108.

- Herbst RS, Heymach JV, Lippman SM: Lung cancer. N Engl J Med 2008;359:1367-1380.

-3 Salama JK, Vokes EE: New radiotherapy and chemoradiotherapy approaches for non-small-cell lung cancer. J Clin Oncol 2013;31:1029-1038.

4 Mittal V, El Rayes T, Narula N, McGraw TE, Altorki NK, Barcellos-Hoff MH: The microenvironment of lung cancer and therapeutic implications; in Lung Cancer and Personalized Medicine: Novel Therapies and Clinical Management (eds): Advances in Experimental Medicine and Biology. Springer, 2016, vol. 890, pp. 75-110.

5 Barker HE, Paget JT, Khan AA, Harrington KJ: The tumour microenvironment after radiotherapy: mechanisms of resistance and recurrence. Nat Rev Cancer 2015;15:409.

6 Blackhall F, Faivre-Finn C: Treatment of limited small cell lung cancer: an old or new challenge? Curr Opin Oncol 2011;23:158-162.

7 Yeung KT, Yang J: Epithelial-mesenchymal transition in tumor metastasis. Mol Oncol 2017;11:28-39.

8 Marcucci F, Stassi G, De Maria R: Epithelial-mesenchymal transition: a new target in anticancer drug discovery. Nat Rev Drug Discov 2016;15:311. 


\section{Cellular Physiology Cell Physiol Biochem 2018;50:721-733 and Biochemistry \begin{tabular}{l|l} 
DOI: 10.1159/000494238 & (c) 2018 The Author(s). Published by S. Karger AG, Basel \\
www.karger.com/cpb
\end{tabular}

9 Sato M, Shames DS, Hasegawa Y: Emerging evidence of epithelial-to-mesenchymal transition in lung carcinogenesis. Respirology 2012;17:1048-1059.

10 Jung JW, Hwang SY, Hwang JS, Oh ES, Park S, Han IO: Ionising radiation induces changes associated with epithelial-mesenchymal transdifferentiation and increased cell motility of A549 lung epithelial cells. Eur J Cancer 2007;43:1214-1224.

11 Liu W, Huang Y-J, Liu C, Yang Y-Y, Liu H, Cui J-G, Cheng Y, Gao F, Cai J-M, Li B-L: Inhibition of TBK1 attenuates radiation-induced epithelial-mesenchymal transition of A549 human lung cancer cells via activation of GSK-3 $\beta$ and repression of ZEB1. Lab Invest 2014;94:362-370.

12 Lin X, Duan X, Liang Y-Y, Su Y, Wrighton KH, Long J, Hu M, Davis CM, Wang J, Brunicardi FC: PPM1A functions as a Smad phosphatase to terminate TGF $\beta$ signaling. Cell 2006;125:915-928.

$\checkmark 13$ Bourgeois B, Gilquin B, Tellier-Lebegue C, Ostlund C, Wu W, Perez J, El Hage P, Lallemand F, Worman HJ, Zinn-Justin S: Inhibition of TGF-beta signaling at the nuclear envelope: characterization of interactions between MAN1, Smad2 and Smad3, and PPM1A. Sci Signal 2013;6:ra49.

14 Geng J, Fan J, Ouyang Q Zhang X, Zhang X, Yu J, Xu Z, Li Q, Yao X, Liu X: Loss of PPM1A expression enhances invasion and the epithelial-to-mesenchymal transition in bladder cancer by activating the TGF- $\beta /$ Smad signaling pathway. Oncotarget 2014;5:5700.

15 Fiscella M, Zhang H, Fan S, Sakaguchi K, Shen S, Mercer WE, Woude GFV, O'Connor PM, Appella E: Wip1, a novel human protein phosphatase that is induced in response to ionizing radiation in a p53-dependent manner. Proc Natl Acad Sci U S A 1997;94:6048-6053.

16 Hamilton J, Grawenda AM, Bernhard E: Phosphatase inhibition and cell survival after DNA damage induced by radiation. Cancer Biol Ther 2009;8:1577-1586.

17 Lin S, Gregory RI: MicroRNA biogenesis pathways in cancer. Nat Rev Cancer 2015;15:321-333.

18 Yonemori K, Kurahara H, Maemura K, Natsugoe S: MicroRNA in pancreatic cancer. J Hum Genet 2017;62:33.

19 Markou A, Zavridou M, Lianidou ES: MicroRNA signatures as clinical biomarkers in lung cancer. Curr Biomark Find 2015;5:35-45.

20 Goh JN, Loo SY, Datta A, Siveen KS, Yap WN, Cai W, Shin EM, Wang C, Kim JE, Chan M: microRNAs in breast cancer: regulatory roles governing the hallmarks of cancer. Biol Rev Camb Philos Soc 2016;91:409-428.

21 Budhu A, Wang XW: MicroRNAs and gastroenterological cancers. Drug Discov Today Dis 2011;8:e95-e102.

22 Yan D, Ng WL, Zhang X, Wang P, Zhang Z, Mo Y-Y, Mao H, Hao C, Olson JJ, Curran WJ: Targeting DNA-PKcs and ATM with miR-101 sensitizes tumors to radiation. PloS one 2010;5:e11397.

23 Salim H, Akbar N, Zong D, Vaculova A, Lewensohn R, Moshfegh A, Viktorsson K, Zhivotovsky B: miRNA-214 modulates radiotherapy response of non-small cell lung cancer cells through regulation of p38MAPK, apoptosis and senescence. Br J Cancer 2012;107:1361-1373.

24 Oh J-S, Kim J-J, Byun J-Y, Kim I-A: Lin28-let7 modulates radiosensitivity of human cancer cells with activation of K-Ras. Int J Radiat Oncol Biol Phys 2010;76:5-8.

25 Shin S, Cha HJ, Lee E-M, Lee S-J, Seo S-K, Jin H-O, Park I-C, Jin Y-W, An S: Alteration of miRNA profiles by ionizing radiation in A549 human non-small cell lung cancer cells. Int J Oncol 2009;35:81-86.

26 Lu J, Zhong Y, Lin X, Lin Z, Chen Z, Wu X, Wang N, Lin S: Jiawei Maxing Shigan Decoction (JMSD) attenuates radiation-induced epithelial-mesenchymal transition of primary rat type II alveolar epithelial cells. Int J Clin Exp Med 2017;10:16292-16300.

-27 Lu J, Zhong Y, Lin Z, Lin X, Chen Z, Wu X, Wang N, Zhang H, Huang S, Zhu Y: Baicalin alleviates radiationinduced epithelial-mesenchymal transition of primary type II alveolar epithelial cells via TGF- $\beta$ and ERK/ GSK3 $\beta$ signaling pathways. Biomed Pharmacother 2017;95:1219-1224.

-28 Livak KJ, Schmittgen TD: Analysis of relative gene expression data using real-time quantitative PCR and the 2- $\Delta \Delta$ CT method. Methods 2001;25:402-408.

29 Moffat J, Grueneberg DA, Yang X, Kim SY, Kloepfer AM, Hinkle G, Piqani B, Eisenhaure TM, Luo B, Grenier JK, Carpenter AE, Foo SY, Stewart SA, Stockwell BR, Hacohen N, Hahn WC, Lander ES, Sabatini DM, Root DE: A lentiviral RNAi library for human and mouse genes applied to an arrayed viral high-content screen. Cell 2006;124:1283-1298.

30 Yu S-L, Chen H-Y, Chang G-C, Chen C-Y, Chen H-W, Singh S, Cheng C-L, Yu C-J, Lee Y-C, Chen H-S: MicroRNA signature predicts survival and relapse in lung cancer. Cancer cell 2008;13:48-57.

-31 Arora H, Qureshi R, Jin S, Park A-K, Park W-Y: miR-9 and let-7g enhance the sensitivity to ionizing radiation by suppression of NFKB1. Exp Mol Med 2011;43:298-304. 


\section{Cellular Physiology Cell Physiol Biochem 2018;50:721-733

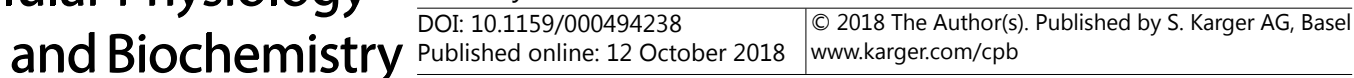

Lu et al.: EMT via miR3591-5p/USP33/PPM1A

-32 Grosso S, Doyen J, Parks S, Bertero T, Paye A, Cardinaud B, Gounon P, Lacas-Gervais S, Noël A, Pouyssegur J: MiR-210 promotes a hypoxic phenotype and increases radioresistance in human lung cancer cell lines. Cell Death Dis 2013;4:e544.

-33 Yang W, Wei J, Sun T, Liu F: Effects of knockdown of miR-210 in combination with ionizing radiation on human hepatoma xenograft in nude mice. Radiat Oncol 2013;8:102.

34 Yang W, Sun T, Cao J, Liu F, Tian Y, Zhu W: Downregulation of miR-210 expression inhibits proliferation, induces apoptosis and enhances radiosensitivity in hypoxic human hepatoma cells in vitro. Exp Cell Res 2012;318:944-954.

-35 Lynam-Lennon N, Reynolds JV, Marignol L, Sheils OM, Pidgeon GP, Maher SG: MicroRNA-31 modulates tumour sensitivity to radiation in oesophageal adenocarcinoma. J Mol Med 2012;90:1449-1458.

36 Dong L, Li Y, Han C, Wang X, She L, Zhang H: miRNA microarray reveals specific expression in the peripheral blood of glioblastoma patients. Int J Oncol 2014;45:746-756.

37 Ninomiya M, Kondo Y, Kimura O, Funayama R, Nagashima T, Kogure T, Morosawa T, Tanaka Y, Nakayama $\mathrm{K}$, Shimosegawa T: The expression of miR-125b-5p is increased in the serum of patients with chronic hepatitis B infection and inhibits the detection of hepatitis B virus surface antigen. J Viral Hepat 2016;23:330-339.

38 Huang Z, Wen P, Kong R, Cheng H, Zhang B, Quan C, Bian Z, Chen M, Zhang Z, Chen X: USP33 mediates Slit Robo signaling in inhibiting colorectal cancer cell migration. Int J Cancer 2015;136:1792-1802.

-39 Yuasa-Kawada J, Kinoshita-Kawada M, Rao Y, Wu JY: Deubiquitinating enzyme USP33/VDU1 is required for Slit signaling in inhibiting breast cancer cell migration. Proc Natl Acad Sci U S A 2009;106:14530-14535.

40 Wen P, Kong R, Liu J, Zhu L, Chen X, Li X, Nie Y, Wu K, Wu JY: USP33, a new player in lung cancer, mediates Slit-Robo signaling. Protein Cell 2014;5:704-713. 